\title{
Periodontal disease among community-dwelling diabetics
}

\begin{abstract}
Periodontal disease is a chronic inflammatory condition and major cause of tooth loss. Prevalence of periodontal disease increases with age and is closely related to certain systemic diseases such as diabetes. Diabetes is a chronic metabolic disease with inflammation. Well-controlled diabetes will help to improve the periodontal condition. The objective of this investigation was to study the prevalence of periodontal disease in a population of community-dwelling adults attending an all-day diabetes awareness and education event.

Methods: Retrospective study of periodontal disease and its relationship with diabetes. Data were collected from attendees at the Diabetes Expo in Pittsburgh, Pennsylvania, sponsored by the American Diabetes Association. Comprehensive periodontal examinations were performed. Information on age, gender, education, smoking, diabetes, periodontal disease, and oral health were collected and analyzed.
\end{abstract}

Results: 206 individuals participated; 97 respondents had periodontal disease and $147 \mathrm{did}$ not. Ninety-three (93) individuals $(47.9 \%)$ without periodontal disease had diabetes and fifty-four (54) individuals (36.3\%) did not have diabetes ( $\mathrm{p}=0.847$ ); this finding was not significant. Good oral health was important among the people attending this event. Those with good oral health had less diabetes compared to people with poor oral health: this finding was statistically significant $(\mathrm{p}=0.004)$.

Conclusion: Cross-sectional analysis of periodontal disease among community-dwelling diabetics revealed that individuals with diabetes who are aware of their illness and attempt to control their glycemic condition have less chance of having periodontal disease $(\mathrm{p}=0.847)$, with oral health an important, significant factor $(\mathrm{p}=0.004)$. Further prospective research with larger sample sizes is recommended to confirm these findings.

\author{
Volume 7 Issue I - 2017
}

\author{
David C, Pouran Famili \\ Department of Periodontics and Preventive Dentistry, \\ University of Pittsburgh, USA
}

\begin{abstract}
Correspondence: Pouran Famili, Department of Periodontics and Preventive Dentistry, University of Pittsburgh, Basement Level Salk Hall School of Dental Medicine, 350I Terrace Street, Pittsburgh PA I526I, Pennsylvania, USA, Tel 4I2-648-8598, Email pof@pitt.edu
\end{abstract}

Received: December 08, 2016 | Published: April 10, 2017

\section{Introduction}

Periodontitis is a chronic inflammatory disease of the oral cavity, which causes destruction of the periodontium. The periodontium acts as the support mechanism for the teeth and is made up of cementum, the periodontal ligament, and alveolar bone. As the periodontium is destroyed, teeth may become loose and can be lost. The public health impact of periodontal disease is great, with nearly half the U.S. affected by some degree of periodontal disease. ${ }^{1}$ A major risk factor for periodontal disease is diabetes. Diabetes is a metabolic disease where those affected either do not produce insulin or are resistant to its action. An estimated $27.8 \%$ of individuals with diabetes remain undiagnosed. ${ }^{2}$ Among those aware of their diabetic status, a large proportion cannot adequately control their disease. Uncontrolled diabetes can lead to many systemic complications including damage to microvasculature and compromised healing. The connection between diabetes and periodontal disease is well documented in the literature. ${ }^{3-6}$ The systemic effects of diabetes increase susceptibility to periodontal disease, making diabetics an at-risk population for periodontitis. Progressive alveolar bone loss associated with periodontitis, if left untreated, will eventually lead to tooth loss and partial or full edentulism. Decreased masticatory function can have adverse nutritional effects including decreased vegetable intake and high fat, low fiber diets. ${ }^{7}$ Nutritional handicaps such as these are factors in the obesity epidemic in the United States.

\section{Background}

NHANES research shows nearly half of the United States adult population suffers from some type of periodontal disease. ${ }^{1}$ This corresponds to 64.7 million people with periodontitis. Moderate periodontitis is most common, with $30 \%$ of cases classified. Mild and severe periodontitis constitute $8.7 \%$ and $8.5 \%$ of the population, respectively. ${ }^{1}$ Rates of periodontitis were highest in men, non-Asian Hispanics, smokers, adults with less than a high school education, and adults earning below $100 \%$ Federal Poverty Levels. ${ }^{1}$

\section{Systemic disease}

Due to the importance of the host response in the protection from periodontal pathogens, a well-documented relationship exists between certain systemic diseases and susceptibility to periodontitis. The most well defined relationship exists with systemic diseases, which negatively affect neutrophils. The neutrophil is the central element of the host response against invading periodontal pathogens. ${ }^{8}$ Innate or acquired defects in number and/or function of neutrophils reveal the importance of this cell type to the pathogenesis of periodontitis. ${ }^{9}$ Systemic diseases affecting the number of neutrophils include agranulocytosis, cyclic neutropenia, familial benign chronic neutropenia, and systemic lupus erythematosus, among others. Diseases affecting neutrophil function include leukocyte adhesion deficiency Type I and II, Chédiak-Hagashi syndrome, Down's 
syndrome, and Papillon-Lefèvre syndrome. ${ }^{9}$ Though many of these diseases are relatively rare to clinical practice, they often manifest as severe and rapid periodontal breakdown. Another well-understood and well-documented relationship exists between periodontitis and diabetes mellitus; periodontitis has been determined a definitive complication of diabetes. ${ }^{10}$ Various mechanisms attempting to explain this relationship have been proposed and will be discussed in detail in a later section. Less clear relationships, particularly with obesity and osteoporosis, have begun to be elucidated recently. Saito et al. ${ }^{11}$ was the first to describe a potential relationship with obesity, discovering that patients with body-mass indexes $(\mathrm{BMI}) \geq 30$ had a relative risk of 8.6 for periodontitis, compared to those with $\mathrm{BMI} \leq 20$, after adjusting for confounding variables. A plausible mechanism is found in the nature of adipose tissue as a complex and metabolically active endocrine organ, secreting numerous immunomodulatory factors, which may affect the periodontium. ${ }^{12}$ Numerous cross-sectional studies have investigated this potential relationship with varying results; longitudinal studies are needed to further investigate this potential relationship. Mixed results have been obtained when osteoporosis has been investigated as a possible risk factor for periodontal disease. Severe osteoporosis may be associated with less-than-favorable attachment level in periodontal disease. ${ }^{13}$ However, a more recent longitudinal study by Famili et al. ${ }^{14}$ found no such association. ${ }^{14}$

\section{Prevalence}

In the United States, a total 29.1 million people or $9.3 \%$ of the population have diabetes. ${ }^{2}$ The prevalence of diabetes is increasing annually, with older individuals, Native Americans, Hispanics, and non-Hispanic blacks more commonly affected than younger individuals and non-Hispanic whites. ${ }^{2}$ The incidence of diabetes is also increasing, with 1.7 million new cases reported in $2012^{2}$ and 1.9 million new cases reported in $2011^{15}$ compared to 1.3 million cases in 2003 and 800,000 in $1998 .{ }^{16}$ Of the close to 30 million cases in the United States, approximately $90 \%$ are Type II. ${ }^{4}$ The World Health Organization estimates 177 million Type II diabetics worldwide and predicts that number doubling by the year $2030,{ }^{17}$ with the greatest numbers of new diabetics likely to occur in developing and emerging societies, due to the global nutrition crisis rooted dually in hunger and obesity. Most data estimate the prevalence of undiagnosed and diagnosed Type II diabetes approaches twenty $\%{ }^{18}$

\section{Risk factors for diabetes}

The risk factors for Type I diabetes are much less clear than those for Type II. While genetics is known to play a larger role in Type II, the prevalence of Type I diabetes is increased in those with a parent or sibling affected by Type I diabetes. According to the American Diabetes Association, ${ }^{19}$ if the father has Type I diabetes, the odds of the offspring having Type I diabetes are 1 in 17. The odds are less if the mother is affected. If both parents have Type I diabetes, the risk ranges from 1: 10, to 1 in 4 . If either parent suffers from Type II polyglandular autoimmune syndrome, a relatively common condition among Type I diabetics, inherited risk for diabetes in the child is 1 in 2. Importantly, inherited risk alone is not sufficient to cause disease and requires an environmental factor such as diet or infection ${ }^{20}$ for fullblown Type I diabetes to develop. Many hypothesized environmental factors, and their relationship to genetic susceptibility, are still not well understood. Risk factors for Type II diabetes are better understood and well described in the literature. Typically grouped into modifiable and non-modifiable, the constellations of risk factors convey a clear susceptibility to Type II diabetes. Non-modifiable risk factors include age, race, gender, and family history. ${ }^{21}$ The risk of developing diabetes increases significantly with age. ${ }^{22}$ Individuals of African American, Mexican American, and Native American descent are also at higher risk for developing diabetes. Importantly again, the true effect of race is likely confounded by other factors prevalent among these racial groups including obesity, smoking, and hypertension. ${ }^{23}$ However, the magnitude of the differences between ethnic groups when exposed to similar environments implies a significant genetic contribution. ${ }^{21}$ This genetic contribution is further reinforced by a higher prevalence of Type II diabetes in those who have a family history of the disease, though the genes involved in this susceptibility remain unknown. ${ }^{21}$ The most important modifiable risk factor for Type II diabetes is obesity. ${ }^{21}$ More than one-third of American adults and $17 \%$ of youth are overweight or obese. ${ }^{24} \mathrm{~A}$ longitudinal study by Chan et al. ${ }^{25}$ demonstrated obesity to be a powerful predictor of future Type II diabetes. Interventions aimed at reducing obesity have been demonstrated successful in reducing the incidence of Type II diabetes. ${ }^{21}$ While obesity is a personal and global health problem, data suggest ${ }^{26}$ that the body's distribution of fat may be more important than total fat, with visceral or abdominal fat being the best indicator for future disease. In close relation to obesity, physical inactivity is also an important and independent risk factor. Studies have shown ${ }^{27,28}$ that for equivalent levels of obesity, higher levels of physical activity were related with a lower incidence of diabetes. As one might expect when obesity is a significant risk factor, diet and nutrition seem to exert influence on diabetes susceptibility, though the exact role of either is difficult to ascertain. It has been reported ${ }^{29}$ that a high total calorie and low dietary fiber intake, high glycemic load, and a low polyunsaturated-to-saturated fat ratio may predispose to Type II diabetes. Lastly, metabolic syndrome has been shown to increase the risk for both cardiovascular disease and Type II diabetes..$^{30}$ Metabolic syndrome is a common presentation in the United States and consists of at least three of the following clinical findings: abdominal adiposity, low HDL cholesterol, high triglycerides, hypertension, and impaired fasting glucose. In a prospective cohort study with a large sample size, ${ }^{30}$ metabolic syndrome was associated with approximately half of the incident cases of Type II diabetes over an eight-year period, with an age-adjusted relative risk of 6.92 [95\% CI 4.47-10.81]. While clearly many of these risk factors are interdependent, each alone has the ability to decrease the incidence of Type II diabetes if targeted in an intervention. Such interventions should be the focus of public health and medicine to decrease the prevalence of Type II diabetes.

\section{Connection between periodontal disease and diabetes}

The most significant early work establishing relationship between diabetes mellitus and periodontal disease examined rates of both in a homogenous population of non-insulin-dependent diabetic Native Americans (Pima Indians) $)^{31-33}$ with Löe $\mathrm{H}^{10}$ definitively labelling periodontal disease as the sixth complication of diabetes. Grossi and Genco established in the mid-1990s ${ }^{34,35}$ that treatment of periodontal disease would have a beneficial effect on the Type II diabetes condition, reducing glycated hemoglobin; longitudinal research ${ }^{36}$ around the same time showed that poor control of Type II diabetes had a positive association on the severity and extent of periodontal disease. This was further supported by $\mathrm{Kaur}^{5}$, who demonstrated that patients with poorly controlled diabetes are at greater risk for the development of periodontal diseases, as well as for a more severe disease course, than non-diabetics. The bidirectional nature of the relationship between the two diseases suggested by Löe was confirmed by Taylor in $2001^{4}$. By 2005, Genco and Grossi laid groundwork toward defining an inflammatory model linking cytokine production in periodontal disease with the metabolic dysfunction created by obesity in diabetes. ${ }^{37}$ This model was expanded upon 
and, more recently, three major mechanisms explaining the link were presented. ${ }^{3}$ First, diabetic patients display impaired neutrophil activity consisting of a reduced chemotactic response and decreased phagocytic activity. ${ }^{40,41}$ The importance of neutrophil function for periodontal health is well-documented and evidenced by the severe periodontal destruction seen in those suffering from genetic conditions negatively affecting neutrophil number or function. Increasingly in the literature, the diabetic hyper-inflammatory state is being elucidated. While normally pro-inflammatory cytokines such as interleukin-1 $\beta$ play a protective role, over-expression can lead to extensive tissue destruction. ${ }^{3}$ One study comparing the gingival levels of IL-1 $\beta$ in well-controlled and poorly-controlled Type II diabetics revealed significantly higher IL-1 $\beta$ levels in the poorly-controlled group. ${ }^{40} \mathrm{TNF}-\alpha$ has been shown to be increased in obese women, ${ }^{41}$ demonstrating the ability of adipose tissue in Type II diabetics to produce another pro-inflammatory cytokine, possibly contributing to periodontal destruction. The final proposed mechanism centers on the role of advanced glycation end-products (AGE). An indicator of diabetic control over the long-term, these AGE have also been shown to lead to a hyper-inflammatory state, with increased secretion of IL$1, \mathrm{IL}-6$, and TNF- $\alpha^{42}$ It has also been suggested ${ }^{43}$ that AGE correlates with an increased level of destructive reactive oxygen species in the gingiva. AGE not only enhance the inflammatory response but may also reduce bone formation, interfering with remodeling and healing once periodontal treatment is completed. ${ }^{3}$ While research continues to unveil the details of the connection between diabetes and periodontal disease, these proposed mechanisms provide a valid framework for the interaction and underscore the importance of neutrophil function, pro-inflammatory cytokines and bone metabolism.

\section{Materials and methods}

Ours was a retrospective study analyzing periodontal exam data and diabetic health surveys collected as part of the American Diabetes Association Diabetes Expo in Pittsburgh, Pennsylvania, an annual diabetes health education and promotion event. This research was approved by the Institutional Review Board (IRB) of the University of Pittsburgh (IRB\#: PRO15010133). Attendees to the Expo were given the opportunity to have a free complete periodontal examination as one of various health screenings available. Educational pamphlets about the relationship between periodontal disease and diabetes were provided to participants. Information on age, gender, education, smoking, diabetes, periodontal disease and oral health were collected and analyzed.

Data were collected on 206 people; 47 respondents had periodontal disease and 147 did not have periodontal disease, periodontal disease being defined as probing depth $4 \mathrm{~mm}$ or more. Ninety-three (93) individuals [47.9\%] without periodontal disease had diabetes and fifty-four (54) individuals [36.3\%] did not have diabetes [p=0.847 and this was not significant]. Eighty-six (86) individuals [44.3\%] with diabetes and without periodontal disease reported good oral health, versus only fifteen (15) individuals with diabetes and periodontal disease who reported good oral health, a difference $p=0.004$, which was significant. Education was not significant, $p=0.182$. Bleeding upon probing among both diabetics and non-diabetics with periodontal disease was significant, $\mathrm{p}=0.003$.

\section{Discussion}

Various studies have shown the effect of diabetes on periodontal disease, and vice versa. One large study by periodontists and endocrinologists combined found that periodontal disease may contribute to the increased mortality rates among diabetics ${ }^{44}$ while others ${ }^{45}$ have rationalized that advanced periodontitis is associated with higher $\mathrm{HbA1C}$ in both diabetic and non-diabetic individuals, with the effect being greater in patients with progressive periodontitis. Minimal evidence supports any association between periodontal disease and diabetes complications. No studies have considered the level of periodontal disease associated with a likelihood of diabetes complications. Periodontal disease can cause problems for patients with diabetes, in whom the diabetes can be more severe and progress more rapidly than in diabetics without periodontal disease. Control and prevention of periodontal disease may be more important in patients with diabetes. Uncontrolled periodontal disease may affect control of diabetes, and the opposite could be true. It is established that this effect can be modified by good oral hygiene and control of any existing periodontal disease. ${ }^{46,47}$ Results from this research show that not all individuals with diabetes have periodontal disease. In our sample of 206 individuals on whom we performed complete periodontal examinations, ${ }^{47,48}$ had both periodontal disease and diabetes, while 93 individuals with diabetes did not have periodontal disease, a difference was not statistically significant $(\mathrm{p}=0.847)$. Table 1 shows the importance of oral health, specifically that individuals with good oral health have less chance of having diabetes. Periodontal treatment was shown to be a significant factor on the effect of diabetes $(\mathrm{p}=0.000)$. Multivariate logistical regression analysis, Table 2 is shown, controlling for periodontal treatment, gender, education, bleeding upon probing and oral health. The odds of having periodontal disease versus not having periodontal disease among patients with diabetes in our sample was 1.38 , a difference not statistically significant $(\mathrm{p}=0.847)$. Results from our research show that in individuals with diabetes who are aware of their illness and make attempts to control their glycemic condition, prevalence of periodontal disease was low. These results however reflect only cross-sectional analysis with a convenience sample and the finding requires prospective study with larger sample sizes to confirm.

Table I Logistical regression test to show the association between individual covariates and the outcome of interest (gum disease)

\begin{tabular}{|c|c|c|c|c|c|c|}
\hline \multirow{3}{*}{ Characteristics } & \multirow{2}{*}{\multicolumn{2}{|c|}{$\begin{array}{l}\text { Gum disease } \\
{[\mathrm{No}=\mid 47]}\end{array}$}} & \multicolumn{2}{|c|}{ Gum disease } & \multirow{3}{*}{ P-values } & \multirow{3}{*}{ Odds ratio $[95 \% \mathrm{Cl}]$} \\
\hline & & & {$[$ Yes $=4$} & & & \\
\hline & Count & $\%$ & Count & $\%$ & & \\
\hline \multicolumn{7}{|l|}{ Diabetes } \\
\hline Yes & 93 & 47.9 & 29 & 14.9 & \multirow{2}{*}{0.847} & \multirow{2}{*}{$0.935[0.475,1.84]$} \\
\hline No & 54 & 36.3 & 18 & 9.3 & & \\
\hline Missing & 3 & & & & & \\
\hline
\end{tabular}


Table Continued...

\begin{tabular}{|c|c|c|c|c|c|c|}
\hline \multirow{3}{*}{ Characteristics } & \multirow{2}{*}{\multicolumn{2}{|c|}{$\frac{\text { Gum disease }}{[\mathrm{No}=147]}$}} & \multicolumn{2}{|c|}{ Gum disease } & \multirow{3}{*}{ P-values } & \multirow{3}{*}{ Odds ratio $[95 \% \mathrm{Cl}]$} \\
\hline & & & {$[$ Yes $=4$} & & & \\
\hline & Count & $\%$ & Count & $\%$ & & \\
\hline \multicolumn{7}{|l|}{ Oral health } \\
\hline Good & 86 & 44.3 & 15 & 7.7 & \multirow{4}{*}{0.004} & \multirow{4}{*}{$0.5 \mathrm{I}[0.320,0.800]$} \\
\hline Fair & 45 & 23.2 & 23 & 11.8 & & \\
\hline Poor & 16 & 8.2 & 9 & 4.6 & & \\
\hline Missing & 12 & & & & & \\
\hline \multicolumn{7}{|l|}{ Gum bleeding } \\
\hline Yes & 45 & 23.2 & 26 & 13.4 & \multirow{2}{*}{0.003} & \multirow{2}{*}{$2.8[1.43,5.50]$} \\
\hline No & 102 & 52.6 & 21 & 10.8 & & \\
\hline Missing & 3 & & & & & \\
\hline
\end{tabular}

*Gum treatment, oral health, gum bleeding are statistically important for predicting the outcome variable

Table 2 Multivariate logistical regression

\begin{tabular}{llllll}
\hline Gum disease & Odds ratio & Standard error & $\mathbf{Z}$ value & $\mathbf{P}>|\mathbf{z}|$ & {$[95 \%$ confidence interval] } \\
\hline Anticenter & 2.32 & 1.41 & 1.38 & 0.167 & {$[0.704 .7 .61]$} \\
Aumtcenter & 22 & 12.1 & 5.62 & 0 & {$[7.49,64.6]$} \\
Gender & 1.02 & 0.454 & 0.04 & 0.969 & {$[0.425,2.44]$} \\
Education & 0.71 & 0.134 & -1.81 & 0.071 & {$[0.490,1.03]$} \\
Diabetes & 1.38 & 0.616 & 0.72 & 0.471 & {$[0.575,3.31]$} \\
Gumbcenter & 1.77 & 0.77 & 1.31 & 0.19 & {$[0.754,4.15]$} \\
Mouth rinses & 1.3 & 0.255 & 1.34 & 0.18 & {$[0.886,1.91]$} \\
Oral health & 0.512 & 0.151 & -2.26 & 0.024 & {$[0.294,0.918]$} \\
Cons & 1.03 & 1.17 & 0.03 & 0.977 & {$[0.111,9.58]$} \\
\hline
\end{tabular}

*Controlling for Gum treatment, Antibiotic, Gender, Education, Gum bleeding, Mouth rinses and oral Health, the odds of having gum disease vs. not having gum disease in patients with diabetes is 1.38

\section{Conclusion}

Retrospective study of periodontal disease among communitydwelling diabetics in this research shows that individuals with diabetes who were aware of their illness and made attempts to control their glycemic condition had less chance of having periodontal disease $(p=0.847)$ and overall better oral health $(p=0.004)$. Further prospective research with a larger sample size is necessary to confirm this finding.

\section{Acknowledgments}

None.

\section{Conflicts of interest}

The authors declare there are no conflicts of interest.

\section{References}

1. Tonsgard JH. Clinical manifestations and management of neurofibromatosis type 1. Semin Pediatr Neurol. 2006;13(1):2-7.

2. Eke PI, Dye BA, Wei L, et al. Update on Prevalence of Periodontitis in Adults in the United States: NHANES 2009 to 2012. J Periodontol. 2015;86(5):611-622.
3. National diabetes statistics report: estimates of diabetes and its burden in the United States, Centers for Disease Control and Prevention, Atlanta. 2014.

4. Lakschevitz F, Aboodi G, Tenenbaum H, et al. Diabetes and periodontal diseases: Interplay and links. Curr Diabetes Rev. 2011;7(6):433-439.

5. Taylor GW. Bidirectional interrelationships between diabetes and periodontal diseases: an epidemiological perspective. Ann Periodontol. 2001;6(1):99-112.

6. Kaur G, Holtfreter B, Rathmann W, et al. Association between Type 1 and Type 2 diabetes with periodontal disease and tooth loss. $J$ Clin Periodontol. 2009;36(9):765-774.

7. Tsai C, Hayes C, Taylor GW. Glycemic control of Type 2 diabetes and severe periodontal disease in the US adult population. Community Dent Oral Epidemiol. 2002;30(3):182-192.

8. Nowjack Raymer RE, Sheiham A. Association of edentulism and diet and nutrition in US adults. J Dent Res. 2003;82(2):123-126.

9. Hart TC, Shapira L, Van Dyke TE. Neutrophil defects as risk factors for periodontal diseases. J Periodontol. 1994;65(5 Suppl):521-529.

10. Deas DE, Mackey SA, McDonnell HT. Systemic disease and periodontitis: manifestations of neutrophil dysfunction. Periodontol. 2003;32(1):82 104. 
11. Löe H. Periodontal disease: the sixth complication of diabetes mellitus. Diabetes Care. 1993;16(1):329-334.

12. Saito T, Shimazaki Y, Sakamoto M. Obesity and periodontitis. NEJM. 1998;339(7):482-483.

13. Ritchie CS. Obesity and periodontal disease. Perio. 2007;44(1):154-163.

14. Von Wowern N, Klausen B, Kollerup G. Osteoporosis: a risk factor in periodontal disease. J Periodontol. 1994;65(12):1134-1138.

15. Famili P. Longitudinal study of periodontal disease and edentulism with rates of bone loss in older women. J Periodontol. 2005;76(1):11-15.

16. National diabetes fact sheet: national estimates and general information on diabetes and prediabetes in the United States 2011, Centers for Disease Control and Prevention (CDC). Atlanta, GA: US Department of Health and Human Services; 2011

17. Centers for Disease Control. National diabetes fact sheet 2005. Atlanta, GA: US Department of Health and Human Services.

18. World Health Organization. (2005) The World Health Report 2002 Reducing risks, promoting healthy life. Geneva: World Health Organization 2002.

19. Meneilly GS, Tessier D. Diabetes in elderly adults. The Journals of Gerontology Series A: Biological Sciences and Medical Sciences. 2001;56(1):M5-M13.

20. American Diabetes Association. Genetics of Diabetes. 2014.

21. Laura Dean, Jo McEntyre. The genetic landscape of diabetes. National Center for Biotechnology Information (US). National Library of Medicine, National Institutes of Health, Bethesda, MD. 2004.

22. Alberti K, George MM, Zimmet P, et al. International Diabetes Federation: a consensus on Type 2 diabetes prevention. Diabet Med. 2007;24(5):451463.

23. Holt RIG. Diagnosis, epidemiology and pathogenesis of diabetes mellitus: an update for psychiatrists. Br J Psychiatry. 2004; Suppl 184(47):S55S63.

24. American Heart Association. Understand Your Risk for Diabetes. 2016.

25. Ogden CL, Cynthia L, Margaret D, et al. Prevalence of childhood and adult obesity in the United States, 2011-2012. JAMA. 2014;311(8):806814.

26. Chan JM, Rimm EB, Colditz GA, et al. Obesity, fat distribution, and weight gain as risk factors for clinical diabetes in men. Diabetes Care. 1994;17(9):961-969.

27. Ohlson LO, Larsson B, Svärdsudd K, et al. The influence of body fat distribution on the incidence of diabetes mellitus: 13.5 years of followup of the participants in the study of men born in 1913. Diabetes. 1985;34(10):1055-1058.

28. Hu FB, Li TY, Colditz GA, et al. Television watching and other sedentary behaviors in relation to risk of obesity and Type 2 diabetes mellitus in women. JAMA. 2003;289(14):1785-1791.

29. Hu FB, Leitzmann MF, Stampfer MJ, et al. Physical activity and television watching in relation to risk for Type 2 diabetes mellitus in men. Arch Intern Med. 2001;161(12):1542-1548.

30. Hu FB, Manson JE, Stampfer MJ, et al. Diet, lifestyle, and the risk of Type 2 diabetes mellitus in women. N Engl J Med. 2001;345(11):790-797.

31. Wilson PW, D'Agostino RB, Parise H, et al. Metabolic syndrome as a precursor of cardiovascular disease and Type 2 diabetes mellitus. Circulation. 2005;112(20):3066-3072.
32. Shlossman M, Knowler WC, Pettitt DJ, et al. Type 2 diabetes mellitus and periodontal disease. J Am Dent Assoc. 1990;121(4):532-536.

33. Nelson RG, Shlossman M, Budding LM, et al. Periodontal disease and NIDDM in Pima Indians. Diabetes Care. 1990;13(8):836-840.

34. Emrich LJ, Shlossman M, Genco RJ. Periodontal disease in non-insulindependent diabetes mellitus. J Periodontol. 1991;62(2):123-131.

35. Grossi SG, Skrepcinski FB, DeCaro T, et al. Response to periodontal therapy in diabetics and smokers. $J$ Periodontol. 1996;67(10 Suppl.):1094-1102.

36. Grossi SG, Skrepcinski FB, DeCaro T, et al. Treatment of periodontal disease in diabetics reduces glycated hemoglobin. Journal of Periodontology. 1997;68(8):713-719.

37. Taylor GW, Burt BA, Becker MP, et al. Glycemic control and alveolar bone loss progression in Type 2 diabetes. Ann Periodontol. 1998;3(1):30 39 .

38. Genco RJ, Grossi SG, Ho A, et al. A proposed model linking inflammation to obesity, diabetes, and periodontal infections. J Periodontol. 2005;76(11 Suppl):2075-2084.

39. Gursoy UK, Marakoglu I, Ostop AY. Relationship between neutrophil functions and severityof periodontitis in obese and/or Type 2 diabetic chronic periodontitis patients. Quintessence International. 2008;39(6):485-489.

40. Chanchamroen S, Kewcharoenwong C, Susaengrat W, et al. Human polymorphonuclear neutrophil responses to Burkoholderia pseudomallei in healthy and diabetic subjects. Infection and Immunity. 2009;77(1):456463.

41. Cutler CW, Machen RL, Jotwani R, et al. Heightened gingival inflammation and attachment loss in Type II diabetes with hyperlipidemia. J Periodontol. 1999;70(11):1313-1321.

42. Bullo M, Garcia Lorda $\mathrm{P}$, et al. Systemic inflammation, adipose tissue, tumor necrosis factor and leptin expression. Obes Res. 2003;11(4):525531.

43. Goldin A, Beckman JA, Schmidt AM, et al. Advanced glycation end products: sparking the development of diabetic vascular injury. Circulation. 2006;114(6):597-605.

44. Schmidt AM, Weidman E, Lalla E, et al. Advanced glycation end-products (AGEs) induce oxidant stress in the gingiva: a potential mechanism underlying accelerated periodontal disease associated with diabetes. $J$ Periodontal Res. 1996;31(7):508-515.

45. Saremi A, Nelson RG, Tulloch Reid M, et al. Periodontal disease and mortality in Type 2 diabetes. Diabetes Care. 2005;28(1):27-32.

46. Bascones Martínez A, González Febles J, Sanz Esporrin J. Diabetes and periodontal disease. Review of the literature. American Journal of Dentistry. 2014;27(2):63-67.

47. Katz P, Wirthlin MR, Szpunar SM, et al. Epidemiology and prevention of periodontal disease in individuals with diabetes. Diabetes Care. 1991;14(5):375-385.

48. Taylor GW, Borgnakke WS. Periodontal disease: associations with diabetes, glycemic control and complications. Oral Dis. 2008;14(3):191203. 